\title{
High PINCH1 Expression in Human Laryngeal Carcinoma Associates with Poor Prognosis
}

\author{
Georgios Tsinias $\mathbb{D}^{1,2}$ Sofia Nikou, ${ }^{2}$ Theodoros Papadas, ${ }^{1}$ Panagiotis Pitsos $\mathbb{D},{ }^{2}$ \\ Helen Papadaki $\mathbb{D}^{2},{ }^{2}$ and Vasiliki Bravou ${ }^{2}{ }^{2}$ \\ ${ }^{1}$ Department of Otolaryngology, Head and Neck Surgery, University Hospital of Patras, 26504 Patras, Greece \\ ${ }^{2}$ Department of Anatomy, Medical School of Patras, 26504 Patras, Greece \\ Correspondence should be addressed to Vasiliki Bravou; vibra@upatras.gr
}

Received 23 November 2017; Accepted 24 January 2018; Published 20 March 2018

Academic Editor: Matthias B. Stope

Copyright (c) 2018 Georgios Tsinias et al. This is an open access article distributed under the Creative Commons Attribution License, which permits unrestricted use, distribution, and reproduction in any medium, provided the original work is properly cited.

Focal adhesion signaling to actin cytoskeleton is critically implicated in cell migration and cancer invasion and metastasis. Actin-binding proteins cofilin and N-WASP regulate actin filament turnover, and focal adhesion proteins parvins and $\mathrm{PINCH}$ mediate integrin signaling to the actin cytoskeleton. Altered expression of these proteins has been implicated in human cancer. This study addresses their expression and prognostic significance in human laryngeal carcinoma. Protein expressions of cofilin, N-WASP, $\alpha$-parvin, $\beta$-parvin, and PINCH1 were examined by immunohistochemistry in 72 human laryngeal squamous cell carcinomas. Correlations with clinicopathological data and survival were evaluated. All proteins examined were overexpressed in human laryngeal carcinomas compared to adjacent nonneoplastic epithelium. High expression of PINCH1 was associated significantly with high grade, lymph node-positive, and advanced stage disease. Moreover, high PINCH1 expression significantly associated with poor overall and disease-free survival and high cytoplasmic PINCH1 expression was shown by multivariate analysis to independently predict poor overall survival. In conclusion, we provide novel evidence that focal adhesion signaling to actin cytoskeleton is implicated in human laryngeal carcinogenesis and PINCH1 has prognostic significance in the disease.

\section{Introduction}

Laryngeal cancer is the second most common neoplasm of the upper aerodigestive tract [1]. The majority of the cases $(85 \%-95 \%)$ are classified as squamous cell carcinoma. The 5 -year survival for laryngeal squamous cell carcinoma is $60 \%$, and despite the progress in the diagnosis and treatment, survival has not improved much over the years [1]. Since invasion and metastasis account for increased morbidity and mortality, understanding the molecular mechanisms underlying these processes and identifying novel biomarkers could lead to the development of more efficient therapeutic approaches.

Cell migration is crucial in tumor invasion and metastasis and is regulated through actin cytoskeleton reorganization and focal adhesion turnover. Actin reorganization leads to the formation of protrusive structures at the leading edge of cancer cells that mediate migration and invasion through the extracellular matrix (ECM) [2]. Cofilin is an actinbinding protein that regulates actin cytoskeleton dynamics and induces the formation of lamellipodia and invadopodia [1-6]. Cofilin severs actin filaments directly creating new barbed ends and also depolymerises old actin filaments providing free actin monomers available for the next cycle of polymerisation [1-6]. Several studies suggest a significant role of cofilin in cancer cell invasion and metastasis $[2,5,6]$. Increased expression of cofilin has been previously demonstrated in various human cancers including nonsmall cell lung cancer and squamous esophageal carcinoma, and overexpression of cofilin was frequently associated with adverse prognosis and resistance to therapy [7-10].

N-WASP (neural Wiskott-Aldrich syndrome protein) another actin-binding protein is also critically involved in the regulation of actin cytoskeleton dynamics [11]. When 
activated, N-WASP interacts with the Arp $2 / 3$ complex resulting in vigorous actin polymerisation at the leading edge of motile cells and in the formation of filopodia and invadopodia [11]. Several studies implicate N-WASP in cancer progression. Colocalization of N-WASP and Arp- $2 / 3$ has been demonstrated in the invadopodia of aggressive cancer cell lines, and high expression of N-WASP is associated with cancer metastasis [6, 12-15]. Elevated expression of N-WASP and association with aggressive tumor features and reduced survival have been demonstrated in several human malignancies [9, 13-15].

Focal adhesion proteins $\alpha$-parvin (ILKBP/actopaxin), $\beta$ parvin (affixin), and particularly interesting new cysteinehistidine-rich protein $(\mathrm{PINCH})$ provide a link between the ECM and the actin cytoskeleton at integrin adhesion sites [16-25]. Parvins and PINCH bind to integrin-linked kinase (ILK) forming the heterotrimeric ILK-parvin-PINCH (IPP) complex that functions as a protein scaffold and signaling hub with a key role in focal adhesion assembly and integrin signaling [22-24]. Apart from the IPP, parvins and PINCH are engaged in multiple protein interactions and through these adaptor functions they promote actin-cytoskeleton remodeling, cell spreading, and cell migration [16-22]. Parvins and PINCH also regulate cell survival in part through effects on ILK and Akt signaling [20-25]. Increased expression of parvins and PINCH has been reported in human cancer promoting cancer cell migration, invasion, and apoptosis resistance [26-31].

Overall, cofilin, N-WASP, and focal adhesion proteins parvins and PINCH regulate cellular processes critically implicated in cancer progression. However, no previous studies, to the best of our knowledge, have addressed their role in human laryngeal cancer. This study aims to evaluate their expression and prognostic significance in human laryngeal squamous cell carcinoma.

\section{Materials and Methods}

2.1. Tissue Samples. Formalin-fixed, paraffin-embedded tissue samples from 72 patients with primary squamous cell laryngeal carcinoma that underwent total laryngectomy (plus, neck dissection, radiotherapy, and chemotherapy were appropriate) from 1994 to 2007 were obtained from the Department of Pathology, University Hospital of Patras. Cases were revised by expert pathologists (Helen Papadaki and Vasiliki Bravou). Clinical information and survival data were obtained from the records of the Department of Otolaryngology, Head and Neck Surgery, University Hospital of Patras. Two of the cases were women, and 70 were men. Ages ranged from 28 to 89 years old (average $=61.3$, median $=61$ ). The primary tumor was glottic in 43 cases, supraglottic in 27 cases, and subglottic in 2 cases. According to the WHO classification of tumors, 13 (18.1\%) were classified as well differentiated (grade I), 49 $(68.1 \%)$ as moderately differentiated (grade II), and 10 (13.9\%) as poorly differentiated (grade III) [32]. One case (1.4\%) was stage I, 4 cases (5.6\%) were stage II, 25 cases $(34.7 \%)$ were stage III, and 42 cases $(58.3 \%)$ were stage IV according to the TNM Classification of Malignant
Tumours, 7th edition [33]. Cervical lymph nodes (N) were positive in 19 cases $(26.4 \%)$ while $53(73.6 \%)$ cases had no cervical lymph node metastases. All patients were followed up at least for 5 years. The study was performed in accordance with the Helsinki Declarations and the institutional ethical guidelines.

2.2. Immunohistochemistry. Immunohistochemistry was performed using a peroxidase-based polymer as previously described [27]. Primary antibodies used were rabbit polyclonal anti-nonphosphorylated (active) cofilin-1 antibody (PA1-14111, Thermo Scientific, Waltham, MA, USA; 1:200), anti-N-WASP antibody (sc-20770, Santa Cruz Biotechnology Inc., Santa Cruz, CA, USA; 1:200), antiPARVA ( $\alpha$-parvin) antibody (HPA005964, Sigma-Aldrich, St. Luis, USA; $1: 200$ ), anti- $\beta$-parvin antibody (sc-134832, Santa Cruz Biotechnology, CA, USA; 1:400), and mouse monoclonal anti-PINCH-1 (MABT162, EMD Millipore, Billerica, MA, USA; $1: 80$ ). Bound primary antibodies were detected with the Envision ${ }^{\mathrm{TM}}$ detection kit (K5007, DAKO, Hamburg, Germany) and diaminobenzidine (DAB) as the chromogen according to the user's manual. Both positive (colorectal carcinomas) and negative controls (rabbit immunoglobulin fraction, DAKO, REFX0936) were used.

2.3. Immunohistochemical Evaluation. All slides were examined blinded to the case by expert pathologists (Vasiliki Bravou and Helen Papadaki). Cytoplasmic and nuclear expression was evaluated separately. Immunoreactivity was scored on a scale of $0-3$ according to the intensity of staining and percentage of positive cells as previously described [27]. In short, staining intensity was graded as 0 (negative), 1 (weak), 2 (moderate), and 3 (strong). The percentage of positive cells was scored as $0(<1 \%), 1(1-25 \%), 2(26-50 \%), 3$ (51-75\%), and $4(76-100 \%)$. These two scores were multiplied resulting in immunoreactivity (IR) score with values from 0 to 12 [27]. Cases with IR score $=0$ were considered negative, and cases with IR score $>0$ were considered positive. For statistical purposes, cases with IR score $=0-4$ were considered demonstrating low expression, while cases with IR score $=6-12$ were considered demonstrating high expression of the examined protein.

2.4. Statistical Analysis. Statistical analysis was performed with the IBM ${ }^{\circledR}$ SPSS ${ }^{\circledR}$ for Windows, v24 (SPSS Inc., Chicago, IL, USA). Differences in the protein expression levels between groups of clinicopathological parameters were examined with the nonparametric Kruskal-Wallis and Mann-Whitney $U$ tests. The correlation between the expressions of the proteins was examined using the Spearman rank-order correlation test. Overall survival (OS) was defined as the interval from the date of surgery to the death from any cause, while disease-free survival (DFS) was defined as the interval from the date of surgery to the recurrence. Overall and disease-free survivals were analyzed using the Kaplan-Meier method, and differences between subgroups were compared using the log-rank test. Cox proportional hazard univariate and multivariate analysis was performed to identify predictors of survival. Only factors that 


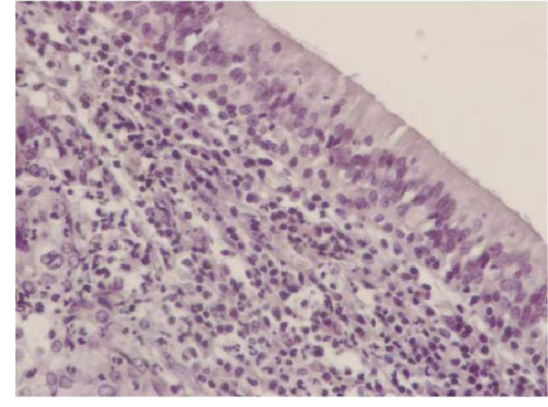

(a)

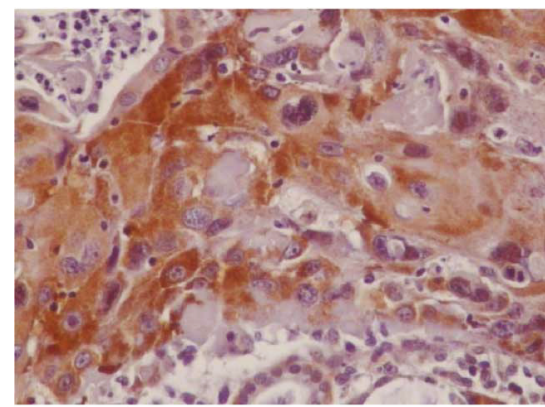

(c)

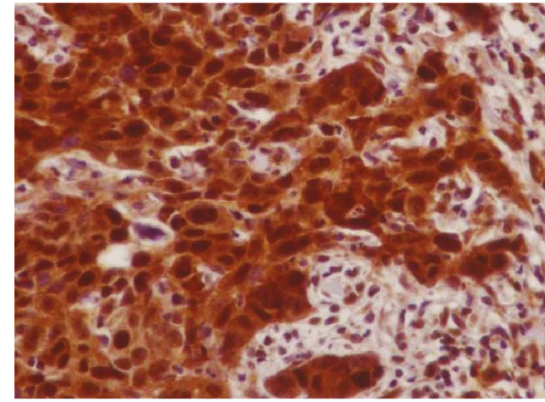

(e)

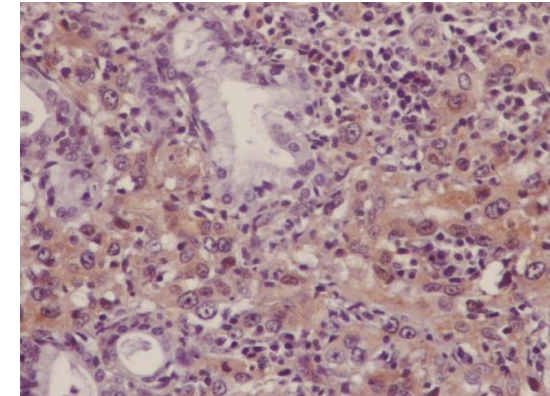

(b)

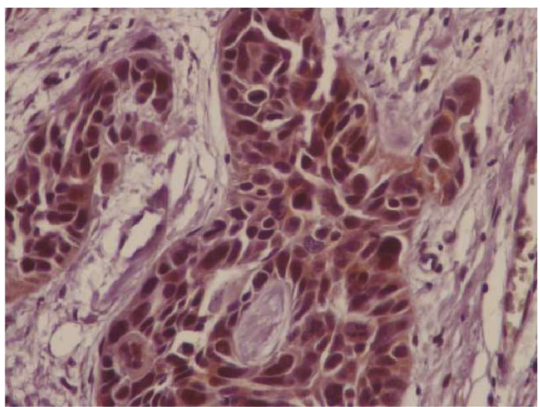

(d)

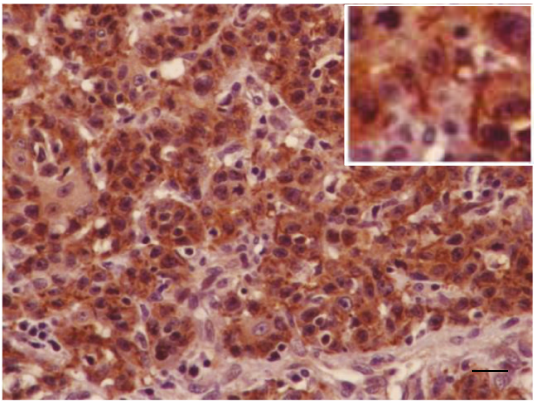

(f)

FIgURE 1: Cofilin immunohistochemical expression in human laryngeal carcinoma. (a) Negative cofilin expression in the adjacent nonneoplastic epithelium. (b) A case of laryngeal carcinoma with low cofilin expression. Representative cases of laryngeal carcinomas with high cytoplasmic (c), nuclear (d), or cytoplasmic and nuclear cofilin expression (e). (f) A case of laryngeal carcinoma with cofilin rods. Bar corresponds to $50 \mu \mathrm{m}(\times 400)$.

showed significance by univariate analysis were included in multivariate analysis. $p$ values $<0.05$ were considered statistically significant.

\section{Results}

3.1. Increased Expression of Actin-Binding Proteins Cofilin and $\mathrm{N}$-WASP in Human Laryngeal Carcinoma. Immunoreactivity for nonphosphorylated (active) cofilin in adjacent nonneoplastic laryngeal epithelium was negative or weakly nuclear (Figure 1(a)). In carcinomas, positive immunohistochemical expression of cofilin was found in 62/72 (86.1\%) cases. Immunoreactivity for cofilin was observed in the cytoplasm and/or the nucleus of cancer cells (Figure 1). Cytoplasmic expression of cofilin was found in 54/72 (75\%) cases, while nuclear expression was found in 41/72 (56.9\%) cases. Occasionally, in $5 / 72(6.9 \%)$ cases, staining for cofilin was rod-like (Figure $1(f)$ ). There was no statistically significant association of cofilin with any of the clinical and pathological parameters under evaluation (age, tumor location, grade, nodal metastases, or stage) (Table 1).

Adjacent nonneoplastic laryngeal epithelium showed negative or weak staining for N-WASP, while positive immunohistochemical expression of N-WASP was found in 67/72 (93.1\%) cases. Expression of N-WASP was mainly cytoplasmic while nuclear staining was observed in 7/72 (9.7\%) cases (Figure 2). Expression of cytoplasmic N-WASP significantly differed among grade groups with high grade tumors showing lower expression of N-WASP $(p=0.032)$. No other statistical significant association of N-WASP with the clinical and pathological parameters under evaluation was found (Table 1). Also, nuclear N-WASP showed no significant associations in our samples.

Survival analysis with the Kaplan-Meier and log-rank test showed no significant association of cofilin or N-WASP expression with OS or DFS. 
TABLE 1: Expression of actin-binding proteins cofilin and N-WASP in human laryngeal cancer in relation to clinical and pathological parameters.

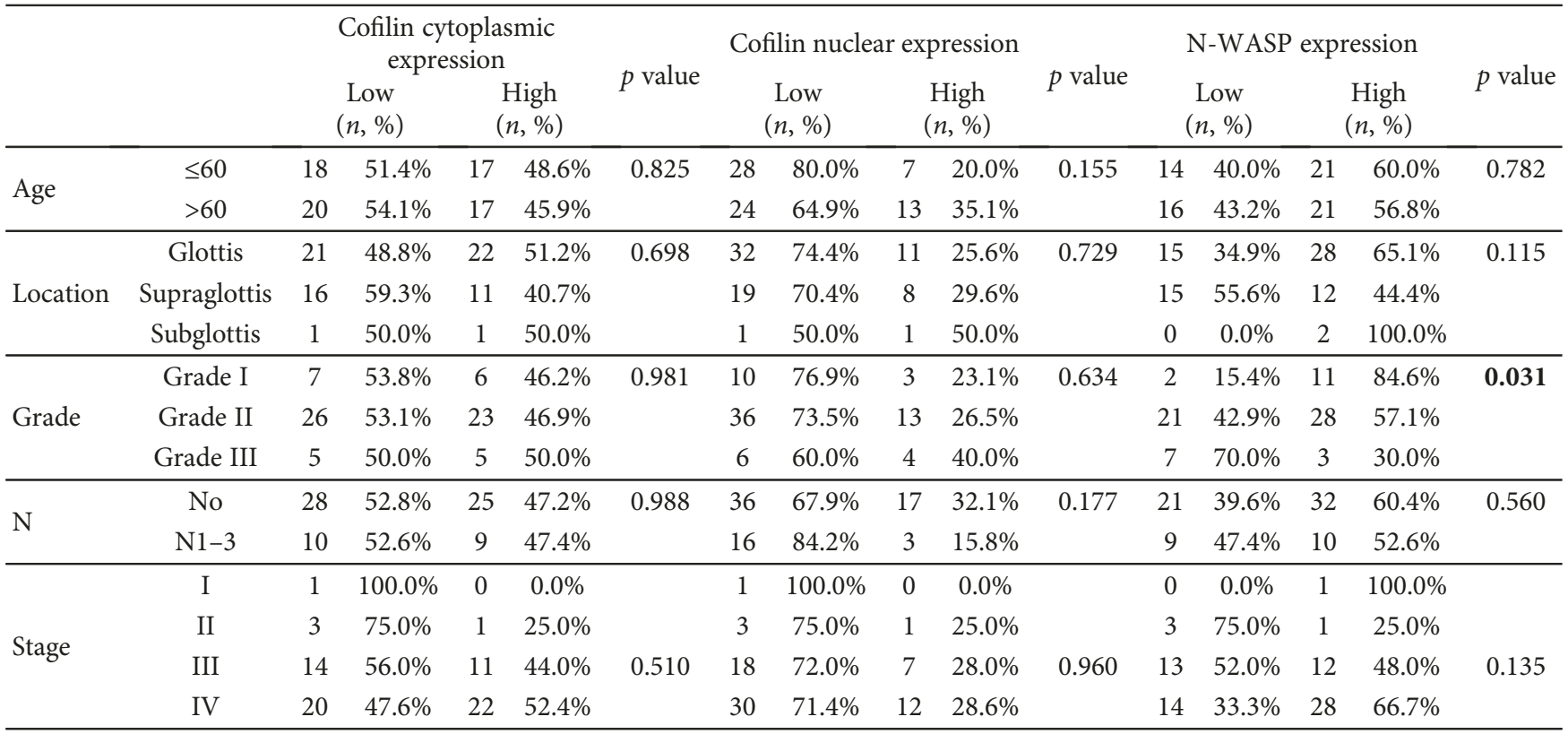

Statistical analyses were performed by nonparametric tests, and $p<0.05$ was considered statistically significant. Lymph node (N) metastasis and stage were determined based on TNM, 7th edition [33].

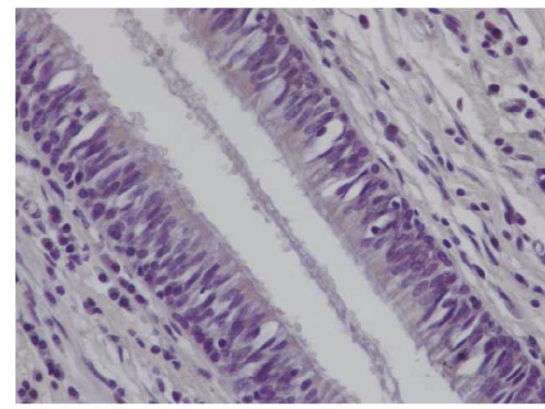

(a)

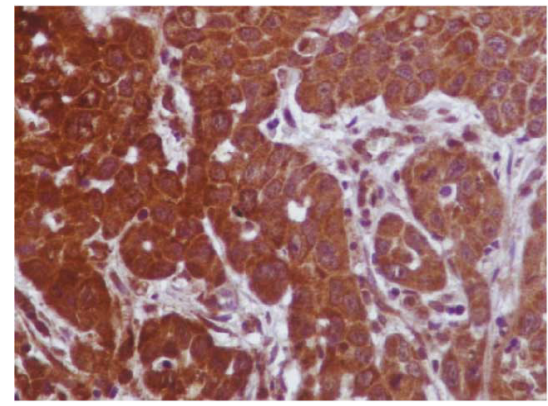

(c)

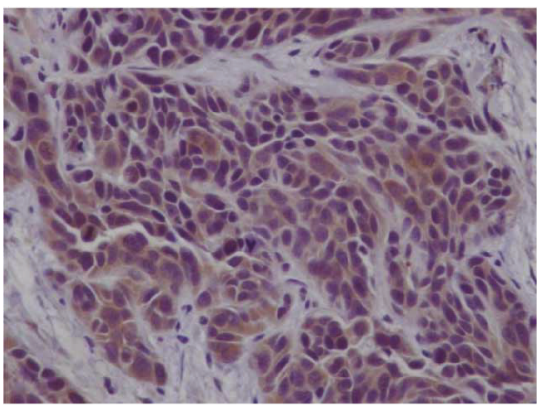

(b)

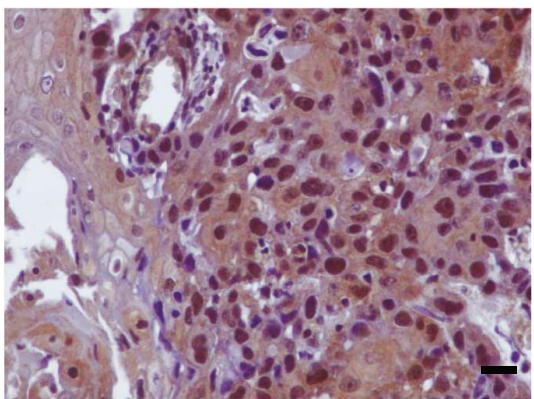

(d)

FIGURE 2: N-WASP immunohistochemical expression in human laryngeal carcinoma. (a) Negative N-WASP expression in the adjacent nonneoplastic epithelium. (b) A case of laryngeal carcinoma with low N-WASP expression. (c) Representative case of laryngeal carcinoma with high cytoplasmic N-WASP expression. (d) Nuclear expression of N-WASP in a case of laryngeal carcinoma. Bar corresponds to $50 \mu \mathrm{m}(\times 400)$.

3.2. Focal Adhesion Proteins $\alpha$ - and $\beta$-Parvin Are Overexpressed in Human Laryngeal Cancer. Epithelial cells of adjacent nonneoplastic tissue demonstrated negative or very weak immunoreactivity for $\alpha$ - and $\beta$-parvin (Figures 3(a) and 3(b)). Positive expression of $\alpha$ - and $\beta$-parvin in tumor cells was noted in 60/72 (83.3\%) and 68/72 (94.4\%) cases of 
$\alpha$-Parvin

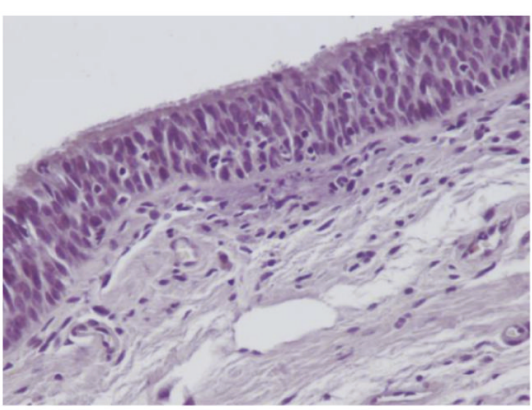

(a)

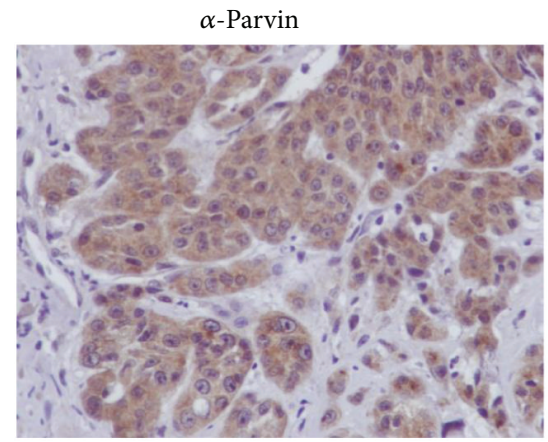

(c)

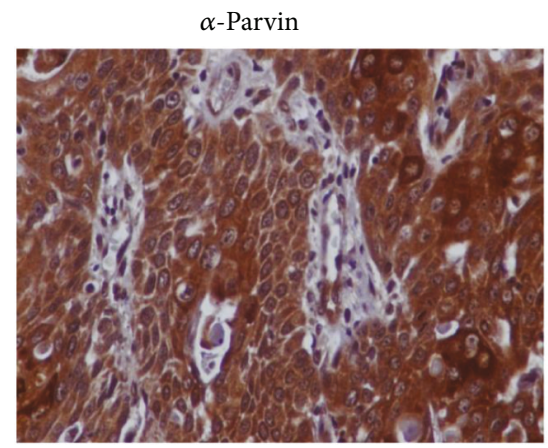

(e)

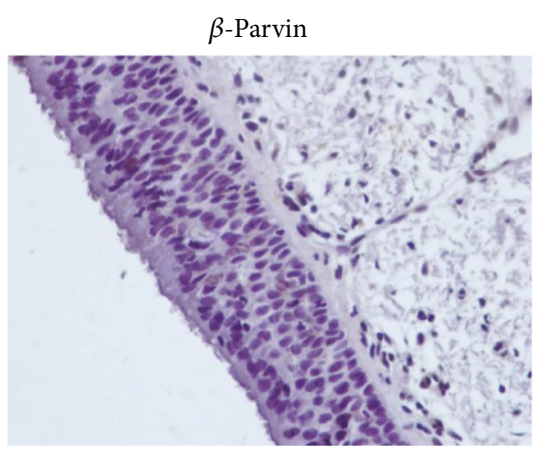

(b)

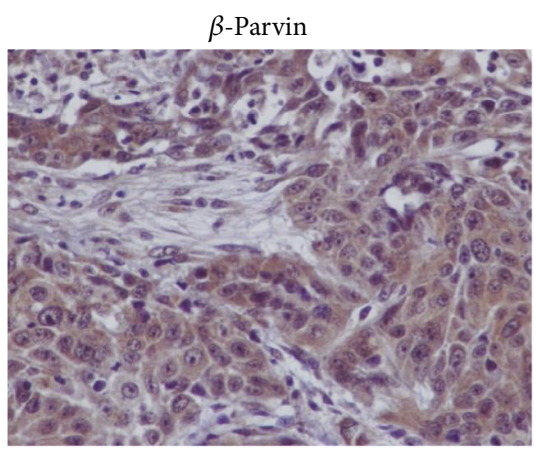

(d)

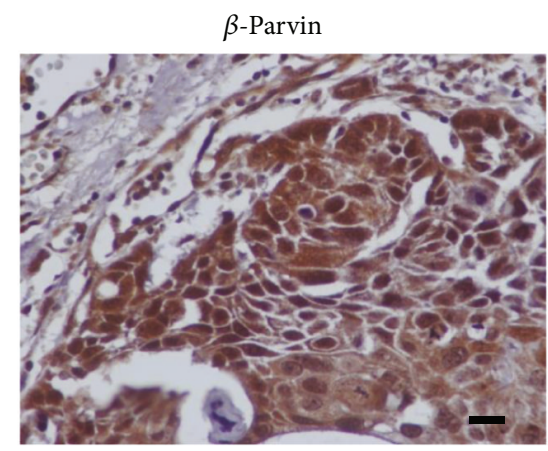

(f)

FIgURE 3: Expression of parvins by immunohistochemistry in human laryngeal carcinoma. (a, b) Negative expression in the adjacent nonneoplastic epithelium. (c, d) Representative cases of laryngeal carcinomas with low expression of parvins. (e, f) Representative cases of laryngeal carcinomas with high expression of $\alpha$-parvin (e, cytoplasmic) and $\beta$-parvin (f, cytoplasmic and nuclear). Bar corresponds to $50 \mu \mathrm{m}(\times 400)$.

laryngeal squamous carcinoma, respectively. Immunostaining of parvins was mainly cytoplasmic while a few cases $(6 /$ $72,8.3 \%)$ showed cytoplasmic and nuclear staining for $\beta$ parvin (Figure 3). No statistically significant association of $\alpha$ - or $\beta$-parvin expression with clinicopathological parameters was found (Table 2). Survival analysis with the KaplanMeier and log-rank test showed no significant association of parvin expression with patient survival. Immunohistochemical expression of focal adhesion proteins $\alpha$ - and $\beta$-parvin significantly and positively correlated with cytoplasmic expression of cofilin $(p=0.034$ and $r=0.251$ for $\alpha$-parvin; $p=0.011$ and $r=0.298$ for $\beta$-parvin), and also, $\beta$-parvin significantly correlated with nuclear cofilin expression $(p=$ $0.009, r=0.304)$.
3.3. Expression of PINCH1 Is a Poor Prognostic Factor in Human Laryngeal Cancer. PINCH1 expression in the adjacent nonneoplastic epithelium was negative or very weak, while cytoplasmic and nuclear expression of PINCH1 in tumor cells was detected in 69/72 (95.8\%) and 22/72 (30.6\%) of cases, respectively (Figure 4 ). Cytoplasmic expression of PINCH1 differed significantly among groups, and it was higher in high grade $(p=0.003)$, lymph node-positive $(p=0.004)$, and high stage $(p=0.001$ for stage IV versus stage III) disease. Nuclear expression of PINCH1 was also significantly associated with high tumor grade $(p<0.001)$ (Table 3). No other significant difference was noted in the nuclear expression of PINCH1 among groups of clinicopathological parameters. The Kaplan-Mayer analysis showed a 
TABLE 2: Expression of focal adhesion proteins $\alpha$ - and $\beta$-parvin in human laryngeal cancer in relation to clinical and pathological parameters.

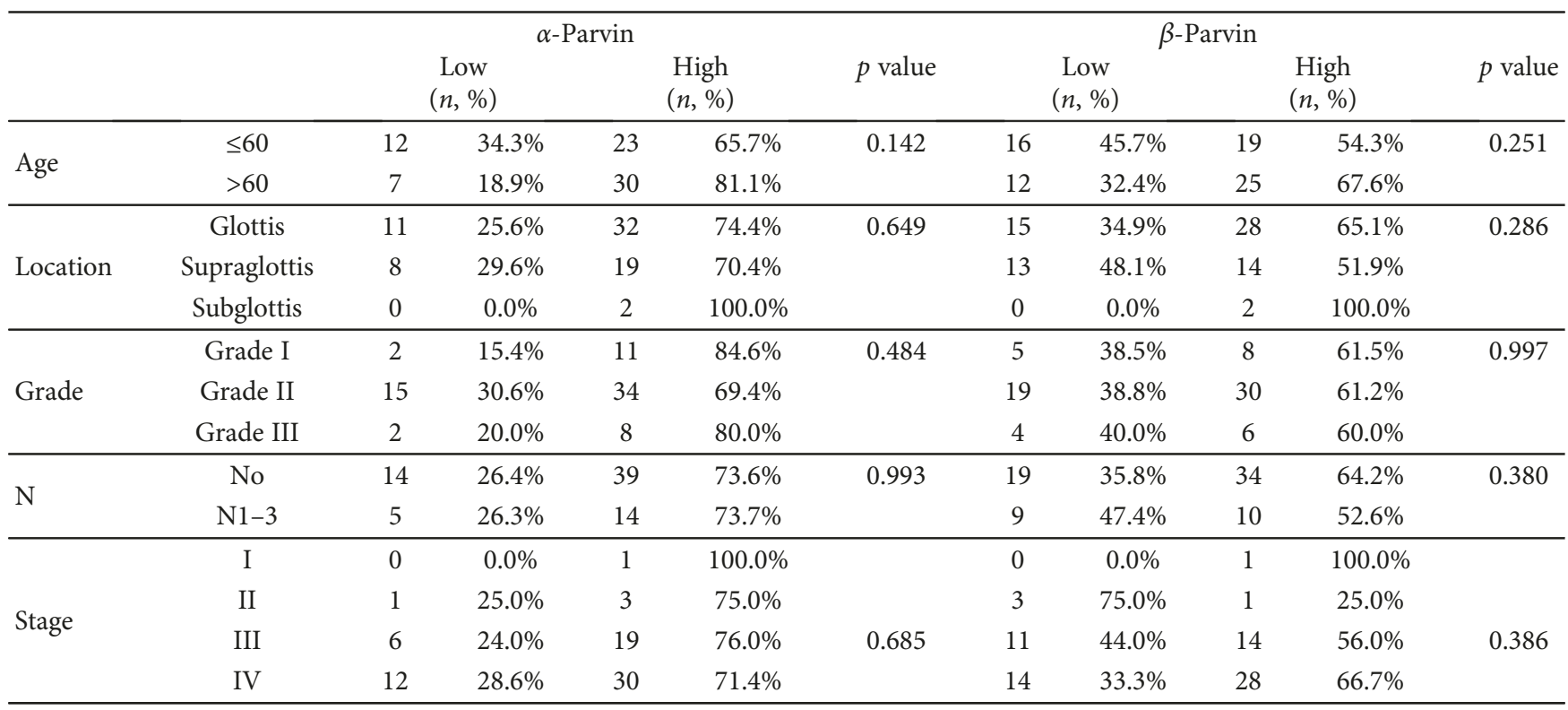

Statistical analyses were performed by nonparametric tests, and $p<0.05$ was considered statistically significant. Lymph node (N) metastasis and stage were determined based on TNM, 7th edition [33].

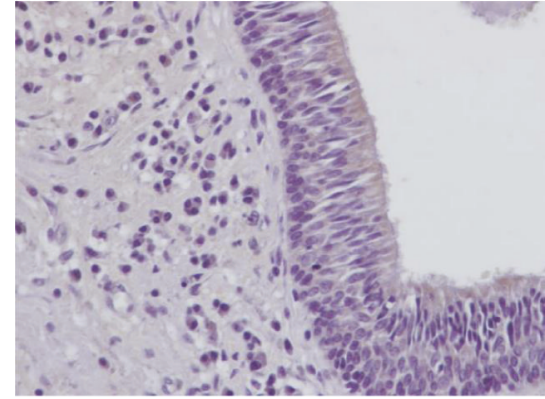

(a)

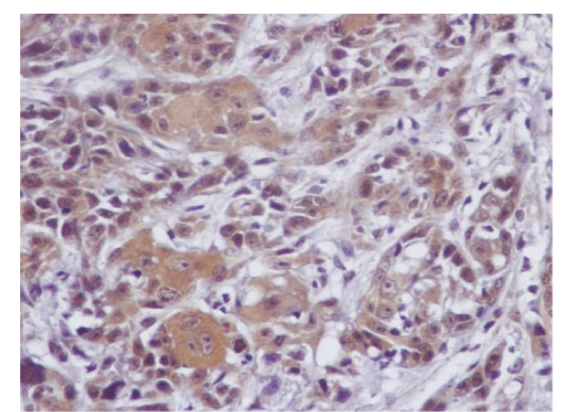

(b)

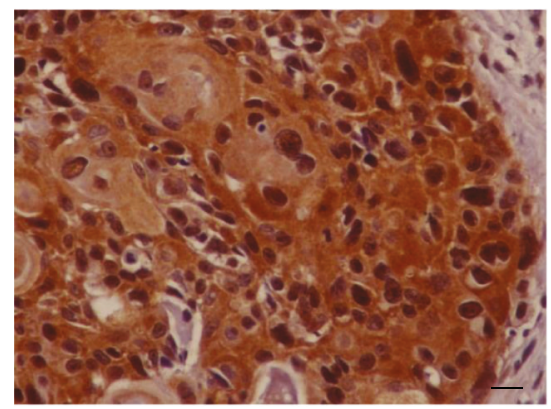

(c)

FIGURE 4: PINCH1 immunohistochemical expression in human laryngeal carcinoma. (a) Negative expression in the adjacent nonneoplastic epithelium. (b) A case of laryngeal carcinoma with low PINCH1 expression. (c) Representative case of laryngeal carcinoma with high PINCH1 expression (cytoplasmic and nuclear). Bar correspond to $50 \mu \mathrm{m}(\times 400)$.

statistically significant association of high cytoplasmic and nuclear PINCH1 expression with reduced OS (28.6 versus 45.4 for low and log-rank $p=0.001$ for cytoplasmic; 17.8 versus 38.4 for low and $p=0.002$ for nuclear). There was also a significant association of high nuclear PINCH1 with reduced DFS (13.3 versus 38.5 for low and log-rank $p=0.016)$
(Figure 5). Univariate analysis showed that grade, $\mathrm{N}$ (lymph node status), and PINCH1 expression are significant predictors of poor overall (both nuclear and cytoplasmic PINCH1) and disease-free survivals (only nuclear PINCH1) (Table 4). Moreover, in multivariate analysis, high cytoplasmic PINCH1 expression was shown to be an independent 
TABLE 3: Expression of PINCH1 in human laryngeal cancer in relation to clinical and pathological parameters.

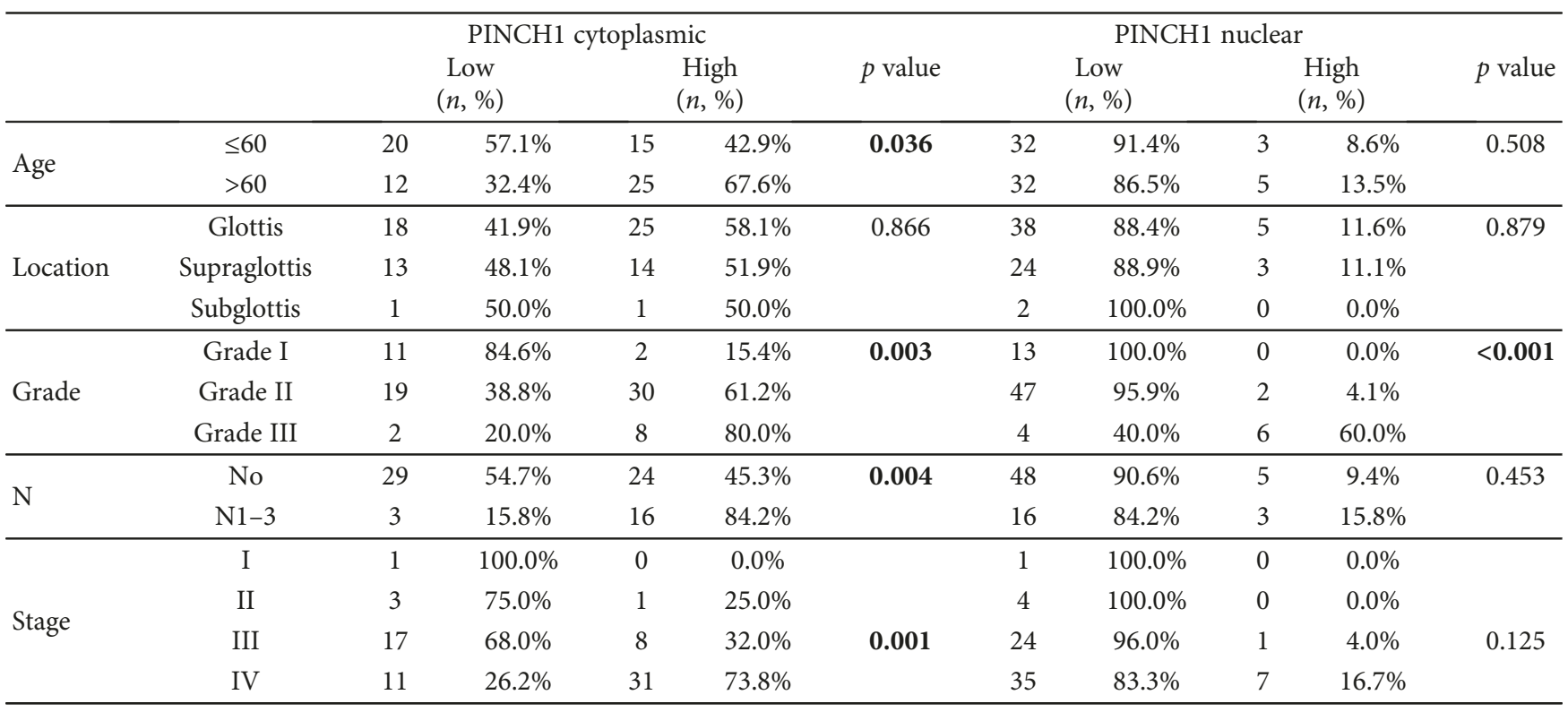

Statistical analyses were performed by nonparametric tests, and $p<0.05$ was considered statistically significant. Lymph node (N) metastasis and stage were determined based on TNM, 7th edition [33].

predictor of poor OS (Table 5). In addition, a strong and positive correlation was found between the cytoplasmic expression of $\beta$-parvin and the cytoplasmic expression of PINCH1 $(p=0.027, r=0.261)$.

\section{Discussion}

Reorganization of the actin cytoskeleton and focal adhesion signaling are fundamental to cancer cell invasion and metastasis $[2,21]$. Actin-binding proteins cofilin and N-WASP and the focal adhesion proteins $\alpha$ - and $\beta$-parvin and PINCH are critically involved in the regulation of actin cytoskeleton dynamics and cell adhesion, survival, and migration, and several studies implicate their altered expression in cancer progression $[2-15,21,22,24-31]$. Here, we originally demonstrate their overexpression in human laryngeal carcinoma and we further show that high expression of PINCH1 is an independent poor prognostic factor.

We showed that active cofilin is overexpressed in laryngeal squamous cell carcinoma suggesting a possible role for cofilin in laryngeal carcinogenesis. In agreement, cofilin is involved in the reorganization of actin cytoskeleton leading to the formation of protrusive structures that enhance motility associated with cancer cell invasion and metastasis [2-6, 34]. Overexpression of cofilin is a feature of cells exhibiting highly invasive and malignant phenotypes [6]. Its expression is elevated in a variety of human cancers, and it is frequently associated with malignant progression [7-10, 34].

Cofilin immunoreactivity in our samples was cytoplasmic and/or nuclear. The cytoplasmic localization of cofilin agrees with its role in the regulation of the actin cytoskeleton [2-5]. In addition, a nuclear localizing signal has been demonstrated in the cofilin sequence and important nuclear functions such as the transport of G-actin into the nucleus to regulate chromatin remodeling and gene transcription and regulation of nuclear architecture have been attributed to cofilin in previous studies [4, 5, 35-39]. In addition, some of our cases showed cytoplasmic and nuclear cofilin rods in tumor cells. Consistently, it has been demonstrated that cofilin-actin accumulations in rod-like patterns form in response to cellular stress and it would be interesting to further explore the significance of nuclear cofilin expression and cofilin rods in human cancer [40].

Many studies support that overexpression of cofilin in human cancer is related to adverse prognostic factors and aggressive biological behavior. Specifically, increased expression of cofilin associated with increased invasion, lymph node metastases, advanced stage, and poor prognosis in several types of cancer [5,7-10,36]. Moreover, several in vitro data show increased cell migration, invasion, and metastasis in cells overexpressing cofilin [5, 34]. However, we found no significant association of cofilin expression with tumor grade, nodal status, stage, and patient survival suggesting that the expression of cofilin in laryngeal cancer does not serve as a prognostic indicator. Nevertheless, this finding needs confirmation with studies that include a larger number of cases (especially early stage) and future research will further clarify the prognostic significance of cofilin overexpression in laryngeal cancer.

N-WASP was also overexpressed in our cohort of tumors suggesting a possible tumorigenic role for N-WASP in laryngeal carcinoma, consistent with reports in other malignancies [9, 13-15]. These findings are in agreement with the known role of N-WASP in the regulation of actin filament turnover and the formation of protrusive structures promoting cell migration and invasion $[6,11,12]$. High N-WASP expression in several types of cancer including squamous oesophageal carcinoma, pancreatic ductal adenocarcinoma, and hepatocellular carcinoma showed correlation with advanced stage, 

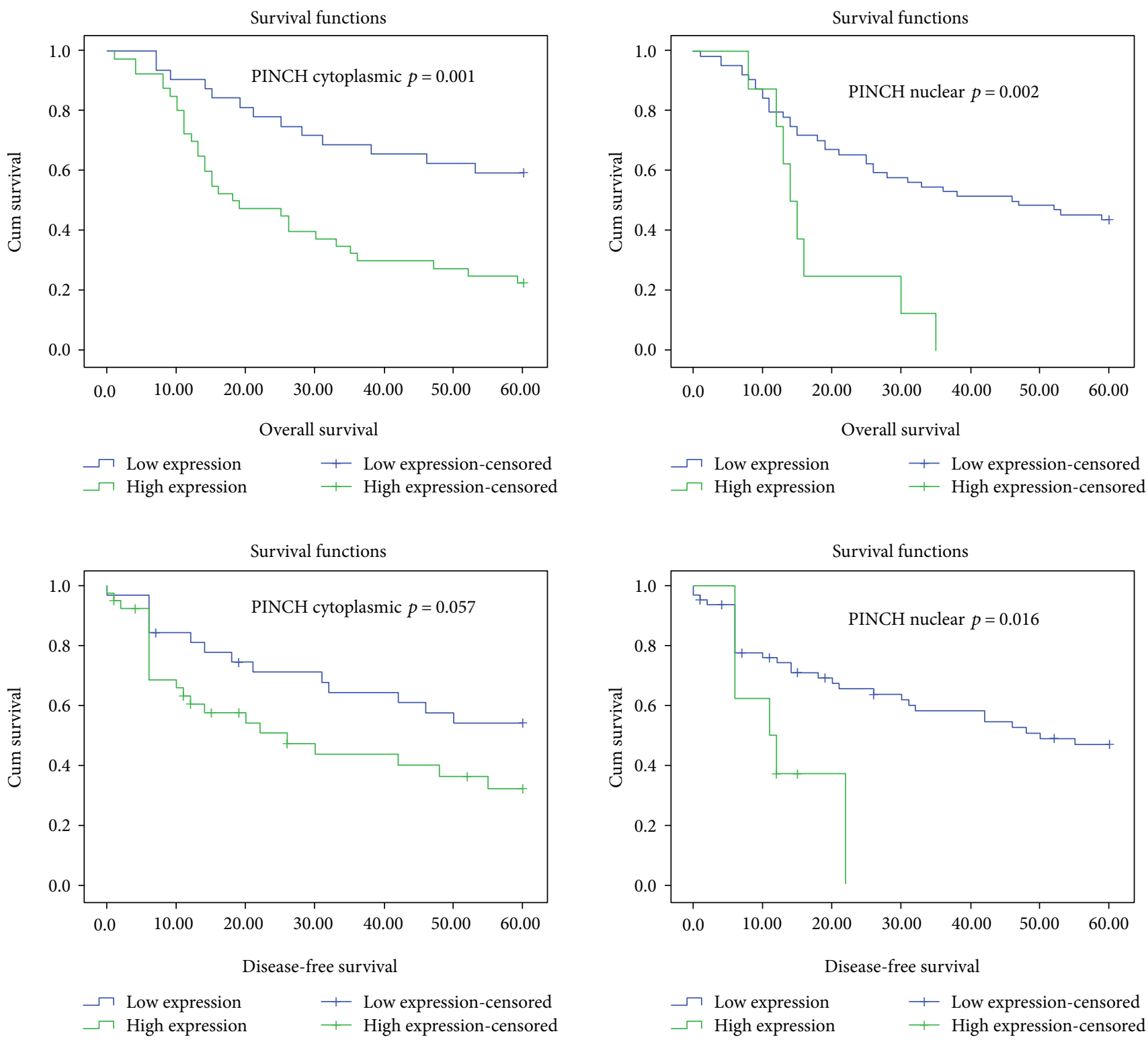

FIGURE 5: Kaplan-Mayer Survival plots. Overall survival and disease-free survival estimates according to cytoplasmic and nuclear PINCH1 expression. Log-rank test; $p$ values $<0.05$ are considered statistically significant.

metastases, and reduced survival rates, suggesting that $\mathrm{N}$ WASP promotes cancer progression and aggressive tumor behavior $[9,14,15]$. However, we surprisingly found that aggressive high grade tumors show lower expression of $\mathrm{N}$ WASP. It should be noted though that there are studies supporting a different role for N-WASP in cancer as low expression of N-WASP associated with metastases and poor prognosis in breast cancer and induction of N-WASP expression reduced breast cancer cell invasiveness [41]. N-WASP also exerts functions in maintaining cell junctions and regulating gene expression [42, 43]. Consistent with studies showing that N-WASP localizes in the nucleus and in nuclear actin complexes, regulating gene transcription, we found nuclear localization of N-WASP in few of our cases $[36,39,43]$. Nevertheless, we found no significant correlations of N-WASP expression with survival suggesting that further evaluation of the prognostic role of N-WASP in human laryngeal cancer is required.

In addition, we demonstrated that focal adhesion proteins $\alpha$ - and $\beta$-parvin are overexpressed in our cohort of tumors although no significant association with adverse prognostic factors was confirmed. Parvins localize at focal adhesions with an important role in integrin signaling and in the regulation of cell adhesion, cell migration, proliferation, and survival [16-18, 21-23]. While $\alpha$-parvin has been shown to promote cancer progression in colorectal, breast, lung, and hepatocellular carcinomas, a controversial role of $\beta$-parvin expression in cancer has been observed in various studies [26-30, 44-46]. Studies in breast and urothelial carcinomas favor a tumor suppressive role of $\beta$-parvin, whereas increased $\beta$-parvin expression has been demonstrated in colorectal cancer, tongue cancer, and chondrosarcomas, 
TABLE 4: Univariate Cox regression analysis for overall and disease-free survival.

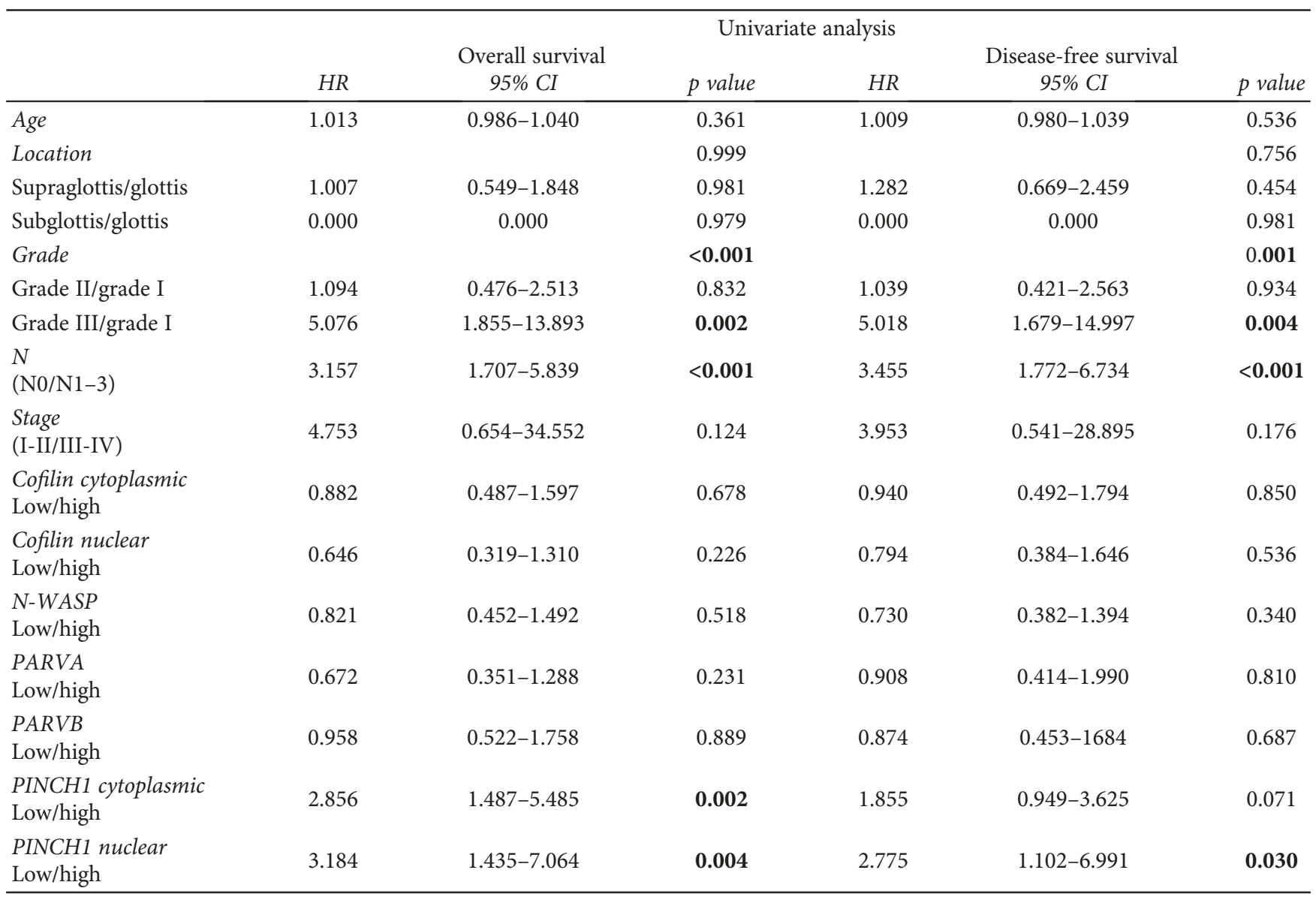

HR: hazard ratio; CI: confidence interval. Significant $p$ values appear in bold.

TABle 5: Multivariate Cox regression analysis for overall and disease-free survival.

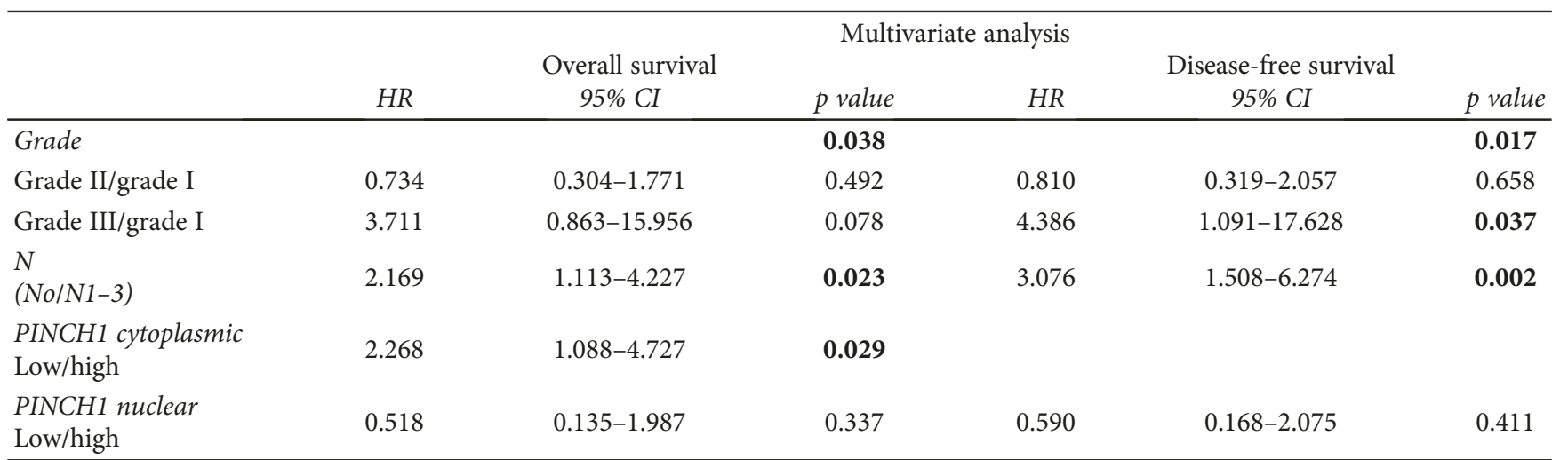

HR: hazards ratio; CI: confidence interval. Significant $p$ values appear in bold. Only parameters that showed a significant difference by univariate analysis were included in the multivariate analysis.

indicating that $\beta$-parvin functions in human cancer may depend on the tissue context [26, 27, 30,44-46]. Our results are consistent with a possible tumorigenic role for both parvins in squamous laryngeal carcinomas. However, further evaluation of their significance in laryngeal cancer in larger cohorts of tumors is needed.
Interestingly, $\alpha$ - and $\beta$-parvin expression correlated with cofilin expression in our samples. Consistently, parvins interact with guanine nucleotide exchange factors (GEFs) and GTPase-activating proteins (GAPs) to promote integrininduced Rho family signaling to the cytoskeleton and regulate lamellipodia formation and cell migration [47-50]. 
Specifically, $\beta$-parvin binds to the GEF factor alpha and beta PIX, which in turn is important for PAK activation and Rho signaling to the actin cytoskeleton, a process involving the LIMK/cofilin pathway [47-49, 51, 52]. $\alpha$-Parvin also binds to the serine/threonine kinase testicular protein kinase 1 (TESK1) a ubiquitously expressed protein reported to regulate actin cytoskeleton dynamics via phosphorylation of cofilin $[53,54]$. Other studies also suggest that the ILK/ $\beta$-parvin/ cofilin pathway mediates invasiveness and metastatic behavior of cancer cells [55].

We also showed for the first time that the focal adhesion protein PINCH1 is overexpressed in human laryngeal carcinoma. Apart from its cytoplasmic localization that is consistent with the function in focal adhesions, PINCH1, as well as $\beta$-parvin, also showed nuclear localization in some of our samples. It is interesting that ILK, their binding partner, localizes at centrosomes and mitotic spindles and regulates mitosis [56]. Both PINCH and $\beta$-parvin have a nuclear localization signal, and although their nuclear functions are yet unknown, an interesting hypothesis that needs to be explored is that they also interact with microtubules, similarly to their partner ILK, regulating mitosis $[17,56,57]$. Importantly, we demonstrated that high PINCH1 expression correlated with aggressive disease and adverse prognosis. Consistent with a tumor-promoting role of PINCH, it has been shown that PINCH interacts at focal adhesions sites with parvins, ILK, Nck1, Rsu-1, and several other partners to promote cell spreading, migration, and apoptosis resistance, features important for tumor cells [19-21, 24, 25]. In further agreement, increased PINCH expression has been demonstrated in tumor-associated stroma at the invasive front in several types of cancer and also high expression of PINCH in esophageal squamous carcinoma correlated with nodal metastases $[24,25,58]$. Also, increased expression of PINCH1 has been reported in colorectal carcinoma compared with a normal colon and high PINCH expression at the invasive front of colorectal cancer as well as in mucinous colorectal adenocarcinoma associated with poor survival [59-64]. Increased PINCH expression in cancer has been also associated with therapy resistance $[59,63,64]$.

In further support to the implication of parvins and PINCH1 in human laryngeal cancer, we have previously shown that ILK, their binding partner, is overexpressed in laryngeal cancer [65]. As already outlined, parvins and PINCH interact with ILK in cells to form a ternary IPP complex $[16,18-21]$. Noteworthy, expression of the individual members of the IPP complex depends on its formation [20]. IPP complex formation has been shown to protect its members from degradation [20]. Also, knockdown of ILK has been shown to reduce levels of parvins in intestinal epithelial cells and expression of $\alpha$ - and $\beta$-parvin in human colorectal cancer positively correlated with levels of ILK $[27,66]$. As our previous study of ILK in laryngeal cancer involved a different tumor cohort, it would be interesting for the reasons stated above to further evaluate ILK levels in this cohort of tumors and examine associations and colocalization of ILK, parvins, and PINCH1 [65]. Different ILK expression levels or preferential formation of an IPP complex containing $\beta$-parvin instead of a-parvin in some tumors may for example account for the higher positivity ratio of $\beta$-parvin, observed in laryngeal tumors, compared to $\alpha$-parvin.

In conclusion, our study suggests that overexpression of actin-binding proteins cofilin, N-WASP, and focal adhesion proteins $\alpha$ - and $\beta$-parvin and PINCH1 is implicated in human laryngeal carcinogenesis. Importantly, we provide novel evidence that high PINCH1 expression is an independent adverse prognostic indicator in human laryngeal cancer.

\section{Conflicts of Interest}

The authors declare no conflict of interest regarding the publication of this paper.

\section{References}

[1] Surveillance, Epidemiology, and End Results (SEER) Program. Research Data (1973-2014)National Cancer Institute, DCCPS, Surveillance Research Programreleased April 2017, based on the November 2016 submission (http://www.seer.cancer.gov).

[2] H. Yamaguchi and J. Condeelis, "Regulation of the actin cytoskeleton in cancer cell migration and invasion," Biochimica et Biophysica Acta (BBA)- Molecular Cell Research, vol. 1773, no. 5, pp. 642-652, 2007.

[3] V. DesMarais, M. Ghosh, R. Eddy, and J. Condeelis, "Cofilin takes the lead," Journal of Cell Science, vol. 118, no. 1, pp. 19-26, 2005.

[4] J. R. Bamburg and B. W. Bernstein, "Roles of ADF/cofilin in actin polymerization and beyond," F1000 Biology Reports, vol. 2, no. 62, 2010.

[5] S. Shishkin, L. Eremina, N. Pashintseva, L. Kovalev, and M. Kovaleva, "Cofilin-1 and other ADF/cofilin superfamily members in human malignant cells," International Journal of Molecular Sciences, vol. 18, no. 12, p. 10, 2017.

[6] H. Yamaguchi, M. Lorenz, S. Kempiak et al., "Molecular mechanisms of invadopodium formation: the role of the N-WASP-Arp2/3 complex pathway and cofilin," The Journal of Cell Biology, vol. 168, no. 3, pp. 441-452, 2005.

[7] M. A. Castro, F. Dal-Pizzol, S. Zdanov et al., "CFL1 expression levels as a prognostic and drug resistance marker in nonsmall cell lung cancer," Cancer, vol. 116, no. 15, pp. 3645-3655, 2010.

[8] C. B. Müller, R. L. de Barros, M. A. Castro et al., "Validation of cofilin-1 as a biomarker in non-small cell lung cancer: application of quantitative method in a retrospective cohort," Journal of Cancer Research and Clinical Oncology, vol. 137, no. 9, pp. 1309-1316, 2011.

[9] W. S. Wang, H. J. Zhong, D. W. Xiao et al., "The expression of CFL1 and N-WASP in esophageal squamous cell carcinoma and its correlation with clinicopathological features," Diseases of the Esophagus, vol. 23, no. 6, pp. 512-521, 2010.

[10] S. Nishimura, H. Tsuda, F. Kataoka et al., "Overexpression of cofilin 1 can predict progression-free survival in patients with epithelial ovarian cancer receiving standard therapy," Human Pathology, vol. 42, no. 4, pp. 516-521, 2011.

[11] T. Takenawa and S. Suetsugu, "The WASP-WAVE protein network: connecting the membrane to the cytoskeleton," Nature Reviews Molecular Cell Biology, vol. 8, no. 1, pp. 3748, 2007.

[12] M. Lorenz, H. Yamaguchi, Y. Wang, R. H. Singer, and J. Condeelis, "Imaging sites of N-wasp activity in lamellipodia 
and invadopodia of carcinoma cells," Current Biology, vol. 14, no. 8, pp. 697-703, 2004.

[13] B. Gligorijevic, J. Wyckoff, H. Yamaguchi, Y. Wang, E. T. Roussos, and J. Condeelis, "N-WASP-mediated invadopodium formation is involved in intravasation and lung metastasis of mammary tumors," Journal of Cell Science, vol. 125, no. 3, pp. 724-734, 2012.

[14] J. C. Guo, J. Li, Y. P. Zhao et al., "N-wasp in pancreatic ductal adenocarcinoma: associations with perineural invasion and poor prognosis," World Journal of Surgery, vol. 38, no. 8, pp. 2126-2131, 2014.

[15] K. M. Jin, M. Lu, F. F. Liu, J. Gu, X. J. Du, and B. C. Xing, "NWASP is highly expressed in hepatocellular carcinoma and associated with poor prognosis," Surgery, vol. 153, no. 4, pp. 518-525, 2013.

[16] S. N. Nikolopoulos and C. E. Turner, "Molecular dissection of actopaxin- integrin-linked kinase- paxillin interactions and their role in subcellular localization," Journal of Biological Chemistry, vol. 277, no. 2, pp. 1568-1575, 2002.

[17] T. M. Olski, A. A. Noegel, and E. Korenbaum, "Parvin, a 42 $\mathrm{kDa}$ focal adhesion protein, related to the alpha-actinin superfamily," Journal of Cell Science, vol. 114, no. 3, pp. 525-538, 2001.

[18] S. Yamaji, A. Suzuki, Y. Sugiyama et al., "A novel integrinlinked kinase-binding protein, affixin, is involved in the early stage of cell-substrate interaction," The Journal of Cell Biology, vol. 153, no. 6, pp. 1251-1264, 2001.

[19] Y. Tu, F. Li, S. Goicoechea, and C. Wu, "The LIM-only protein PINCH directly interacts with integrin-linked kinase and is recruited to integrin-rich sites in spreading cells," Molecular and Cellular Biology, vol. 19, no. 3, pp. 2425-2434, 1999.

[20] T. Fukuda, K. Chen, X. Shi, and C. Wu, "PINCH-1 is an obligate partner of integrin-linked kinase (ILK) functioning in cell shape modulation, motility, and survival," Journal of Biological Chemistry, vol. 278, no. 51, pp. 51324-51333, 2003.

[21] K. R. Legate, E. Montañez, O. Kudlacek, and R. Füssler, "ILK, PINCH and parvin: the tIPP of integrin signalling," Nature Reviews Molecular Cell Biology, vol. 7, no. 1, pp. 20-31, 2006.

[22] J. L. Sepulveda and C. Wu, "The parvins," Cellular and Molecular Life Sciences, vol. 63, no. 1, pp. 25-35, 2006.

[23] Y. Zhang, K. Chen, Y. Tu, and C. Wu, "Distinct roles of two structurally closely related focal adhesion proteins, alphaparvins and beta-parvins, in regulation of cell morphology and survival," Journal of Biological Chemistry, vol. 279, no. 40, pp. 41695-41705, 2004.

[24] H. Xu, H. Cao, and G. Xiao, "Signaling via PINCH: functions, binding partners and implications in human diseases," Gene, vol. 594, no. 1, pp. 10-15, 2016.

[25] J. Kovalevich, B. Tracy, and D. Langford, "PINCH: more than just an adaptor protein in cellular response," Journal of Cellular Physiology, vol. 226, no. 4, pp. 940-947, 2011.

[26] D. J. Papachristou, V. Gkretsi, U. N. Rao et al., "Expression of integrin-linked kinase and its binding partners in chondrosarcoma: association with prognostic significance," European Journal of Cancer, vol. 44, no. 16, pp. 2518-2525, 2008.

[27] V. Bravou, A. Antonacopoulou, S. Papanikolaou et al., "Focal adhesion proteins $\alpha$ - and $\beta$-parvin are overexpressed in human colorectal cancer and correlate with tumor progression,” Cancer Investigation, vol. 33, no. 8, pp. 387-397, 2015.

[28] L. Ng, R. Tung-Ping Poon, S. Yau et al., "Suppression of actopaxin impairs hepatocellular carcinoma metastasis through modulation of cell migration and invasion," Hepatology, vol. 58, no. 2, pp. 667-679, 2013.

[29] M. Ito, M. Hagiyama, T. Mimae et al., “ $\alpha$-Parvin, a pseudopodial constituent, promotes cell motility and is associated with lymph node metastasis of lobular breast carcinoma," Breast Cancer Research and Treatment, vol. 144, no. 1, pp. 59-69, 2014.

[30] A. Eslami, K. Miyaguchi, K. Mogushi et al., " $P A R V B$ overexpression increases cell migration capability and defines high risk for endophytic growth and metastasis in tongue squamous cell carcinoma," British Journal of Cancer, vol. 112, no. 2, pp. 338-344, 2015.

[31] J. Wang-Rodriguez, A. D. Dreilinger, G. M. Alsharabi, and A. Rearden, "The signaling adapter protein pinch is upregulated in the stroma of common cancers, notably at invasive edges," Cancer, vol. 95, no. 6, pp. 1387-1395, 2002.

[32] L. Barnes, J. W. Eveson, P. Reichart, and D. Sidransky, Eds., World Health Organization Classification of Tumours. Pathology and Genetics Head and Neck Tumours, IARC Press, Lyon, 2005.

[33] L. H. Sobin, M. K. Gospodarowicz, and C. H. Wittekind, Eds., UICC International Union Against Cancer, TNM Classification of Malignant Tumors, Wiley-Blackwell, Oxford, UK, 7th edition, 2009.

[34] W. Wang, G. Mouneimne, M. Sidani et al., "The activity status of cofilin is directly related to invasion, intravasation, and metastasis of mammary tumors," The Journal of Cell Biology, vol. 173, no. 3, pp. 395-404, 2006.

[35] F. Matsuzaki, S. Matsumoto, I. Yahara, N. Yonezawa, E. Nishida, and H. Sakai, "Cloning and characterization of porcine brain cofilin cDNA. Cofilin contains the nuclear transport signal sequence," Journal of Biological Chemistry, vol. 263, no. 23, pp. 11564-11568, 1988.

[36] B. Zheng, M. Han, M. Bernier, and J. K. Wen, "Nuclear actin and actin-binding proteins in the regulation of transcription and gene expression," The FEBS Journal, vol. 276, no. 10, pp. 2669-2685, 2009.

[37] E. Castano, V. V. Philimonenko, M. Kahle et al., "Actin complexes in the cell nucleus: new stones in an old field," Histochemistry and Cell Biology, vol. 133, no. 6, pp. 607-626, 2010.

[38] A. Obrdlik and P. Percipalle, "The F-actin severing protein cofilin-1 is required for RNA polymerase II transcription elongation," Nucleus, vol. 2, no. 1, pp. 72-79, 2011.

[39] O. Wiggan, B. Schroder, D. Krapf, J. R. Bamburg, and J. G. DeLuca, "Cofilin regulates nuclear architecture through a myosin-II dependent mechanotransduction module," Scientific Reports, vol. 7, article 40953, 2017.

[40] E. Nishida, K. Iida, N. Yonezawa, S. Koyasu, I. Yahara, and H. Sakai, "Cofilin is a component of intranuclear and cytoplasmic actin rods induced in cultured cells," Proceedings of the National Academy of Sciences of the United States of America, vol. 84, no. 15, pp. 5262-5266, 1987.

[41] T. A. Martin, G. Pereira, G. Watkins, R. E. Mansel, and W. G. Jiang, "N-WASP is a putative tumour suppressor in breast cancer cells, in vitro and in vivo, and is associated with clinical outcome in patients with breast cancer," Clinical \& Experimental Metastasis, vol. 25, no. 2, pp. 97-108, 2008.

[42] C. Rajput, V. Kini, M. Smith et al., "Neural Wiskott-Aldrich syndrome protein (N-WASP)-mediated p120-catenin interaction with Arp2-actin complex stabilizes endothelial adherens junctions," Journal of Biological Chemistry, vol. 288, no. 6, pp. 4241-4250, 2013. 
[43] X. Wu, Y. Yoo, N. N. Okuhama, P. W. Tucker, G. Liu, and J. L. Guan, "Regulation of RNA-polymerase-II-dependent transcription by N-WASP and its nuclear-binding partners," Nature Cell Biology, vol. 8, no. 7, pp. 756-763, 2006.

[44] C. N. Johnstone, P. S. Mongroo, A. S. Rich et al., "Parvin- $\beta$ inhibits breast cancer tumorigenicity and promotes CDK9mediated peroxisome proliferator-activated receptor gamma 1 phosphorylation," Molecular and Cellular Biology, vol. 28, no. 2, pp. 687-704, 2008.

[45] P. S. Mongroo, C. N. Johnstone, I. Naruszewicz et al., “ $\beta$-parvin inhibits integrin-linked kinase signaling and is downregulated in breast cancer," Oncogene, vol. 23, no. 55, pp. 89598970, 2004.

[46] C. F. Wu, K. F. Ng, C. S. Chen et al., "Expression of parvin- $\beta$ is a prognostic factor for patients with urothelial cell carcinoma of the upper urinary tract," British Journal of Cancer, vol. 103, no. 6, pp. 852-860, 2010.

[47] G. Rosenberger, I. Jantke, A. Gal, and K. Kutsche, "Interaction of $\alpha$ PIX (ARHGEF6) with $\beta$-parvin (PARVB) suggests an involvement of $\alpha$ PIX in integrin-mediated signaling," Human Molecular Genetics, vol. 12, no. 2, pp. 155-167, 2003.

[48] W. Mishima, A. Suzuki, S. Yamaji et al., "The first CH domain of affixin activates Cdc42 and Rac1 through alphaPIX, a Cdc42/Rac1-specific guanine nucleotide exchanging factor," Genes to Cells, vol. 9, no. 3, pp. 193-204, 2004.

[49] C. Matsuda, K. Kameyama, A. Suzuki et al., "Affixin activates Rac1 via $\beta$ PIX in C2C12 myoblast," FEBS Letters, vol. 582, no. 8, pp. 1189-1196, 2008.

[50] D. P. LaLonde, M. Grubinger, N. Lamarche-Vane, and C. E. Turner, "CdGAP associates with actopaxin to regulate integrin-dependent changes in cell morphology and motility," Current Biology, vol. 16, no. 14, pp. 1375-1385, 2006.

[51] E. Manser, T. H. Loo, C. G. Koh et al., "PAK kinases are directly coupled to the PIX family of nucleotide exchange factors," Molecular Cell, vol. 1, no. 2, pp. 183-192, 1998.

[52] D. C. Edwards, L. C. Sanders, G. M. Bokoch, and G. N. Gill, "Activation of LIM-kinase by Pak1 couples Rac/Cdc42 GTPase signalling to actin cytoskeletal dynamics," Nature Cell Biology, vol. 1, no. 5, pp. 253-259, 1999.

[53] D. P. LaLonde, M. C. Brown, B. P. Bouverat, and C. E. Turner, "Actopaxin interacts with TESK1 to regulate cell spreading on fibronectin," Journal of Biological Chemistry, vol. 280, no. 22, pp. 21680-21688, 2005.

[54] J. Toshima, J. Y. Toshima, T. Amano, N. Yang, S. Narumiya, and K. Mizuno, "Cofilin phosphorylation by protein kinase testicular protein kinase 1 and its role in integrin-mediated actin reorganization and focal adhesion formation," Molecular Biology of the Cell, vol. 12, no. 4, pp. 1131-1145, 2001.

[55] T. Shibue, M. W. Brooks, and R. A. Weinberg, "An integrinlinked machinery of cytoskeletal regulation that enables experimental tumor initiation and metastatic colonization," Cancer Cell, vol. 24, no. 4, pp. 481-498, 2013.

[56] A. B. Fielding, I. Dobreva, P. C. McDonald, L. J. Foster, and S. Dedhar, "Integrin-linked kinase localizes to the centrosome and regulates mitotic spindle organization," The Journal of Cell Biology, vol. 180, no. 4, pp. 681-689, 2008.

[57] B. J. Martinsen, A. N. Neumann, A. J. Frasier, C. V. H. Baker, C. E. Krull, and J. L. Lohr, "PINCH-1 expression during early avian embryogenesis: implications for neural crest and heart development," Developmental Dynamics, vol. 235, no. 1, pp. 152-162, 2006.
[58] J. T. Zhang, Q. X. Li, D. Wang et al., "Up-regulation of pinch in the stroma of oral squamous cell carcinoma predicts nodal metastasis," Oncology Reports, vol. 14, no. 6, pp. 1519-1522, 2005.

[59] I. Eke, U. Koch, S. Hehlgans et al., "PINCH1 regulates Akt1 activation and enhances radioresistance by inhibiting PP1 $\alpha$," The Journal of Clinical Investigation, vol. 120, no. 7, pp. 2516-2527, 2010.

[60] J. Gao, G. Arbman, A. Rearden, and X. F. Sun, "Stromal staining for pinch is an independent prognostic indicator in colorectal cancer," Neoplasia, vol. 6, no. 6, pp. 796-801, 2004.

[61] A. Holmqvist, J. Gao, B. Holmlund et al., "Pinch is an independent prognostic factor in rectal cancer patients without preoperative radiotherapy - a study in a Swedish rectal cancer trial of preoperative radiotherapy," BMC Cancer, vol. 12 , no. 65 , p. 9 , 2012.

[62] M. J. Wang, J. Ping, Y. Li et al., "Prognostic significance and molecular features of colorectal mucinous adenocarcinomas: a strobe-compliant study," Medicine, vol. 94, no. 51, article e2350, 2015.

[63] A. Bhatia, S. Muthusamy, K. Giridhar, and S. Goel, "Knockdown of PINCH-1 protein sensitizes the estrogen positive breast cancer cells to chemotherapy induced apoptosis," Pathology-Research and Practice, vol. 214, no. 2, pp. 290-295, 2018.

[64] C. Hausmann, A. Temme, N. Cordes, and I. Eke, "ILKAP, ILK and PINCH1 control cell survival of p53-wildtype glioblastoma cells after irradiation," Oncotarget, vol. 6, no. 33, pp. 34592-34605, 2015.

[65] A. K. Goulioumis, V. Bravou, J. Varakis, P. Goumas, and H. Papadaki, "Integrin-linked kinase cytoplasmic and nuclear expression in laryngeal carcinomas," Virchows Archiv, vol. 453, no. 5, pp. 511-519, 2008.

[66] D. Gagné, J. F. Groulx, Y. D. Benoit et al., "Integrin-linked kinase regulates migration and proliferation of human intestinal cells under a fibronectin-dependent mechanism," Journal of Cellular Physiology, vol. 222, no. 2, pp. 387-400, 2010. 


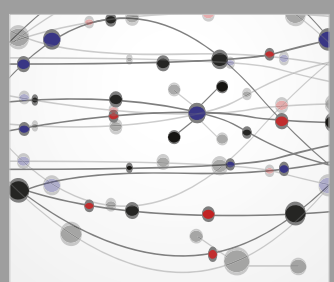

The Scientific World Journal
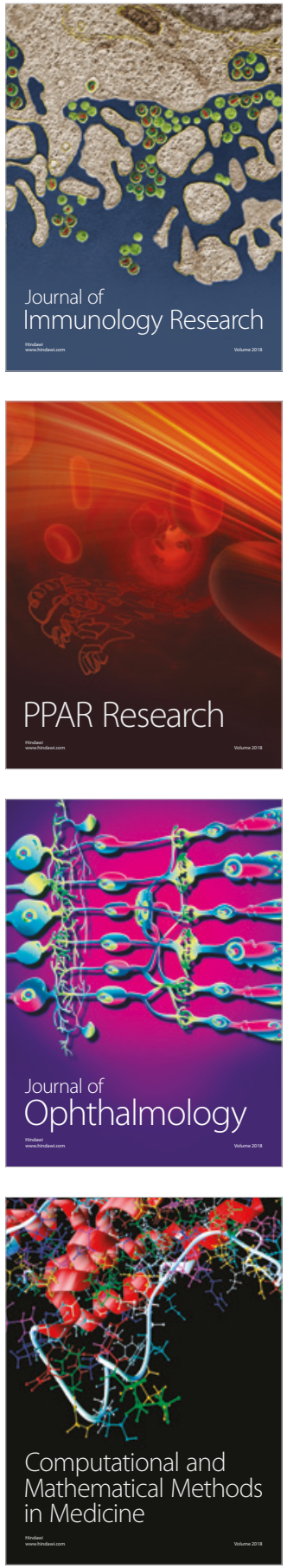

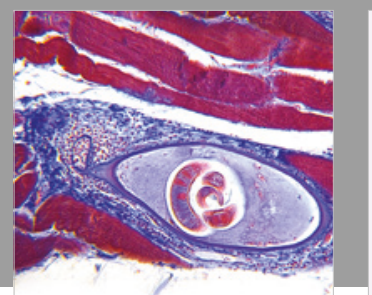

Gastroenterology Research and Practice

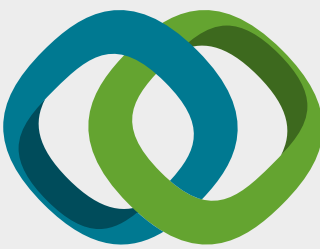

\section{Hindawi}

Submit your manuscripts at

www.hindawi.com
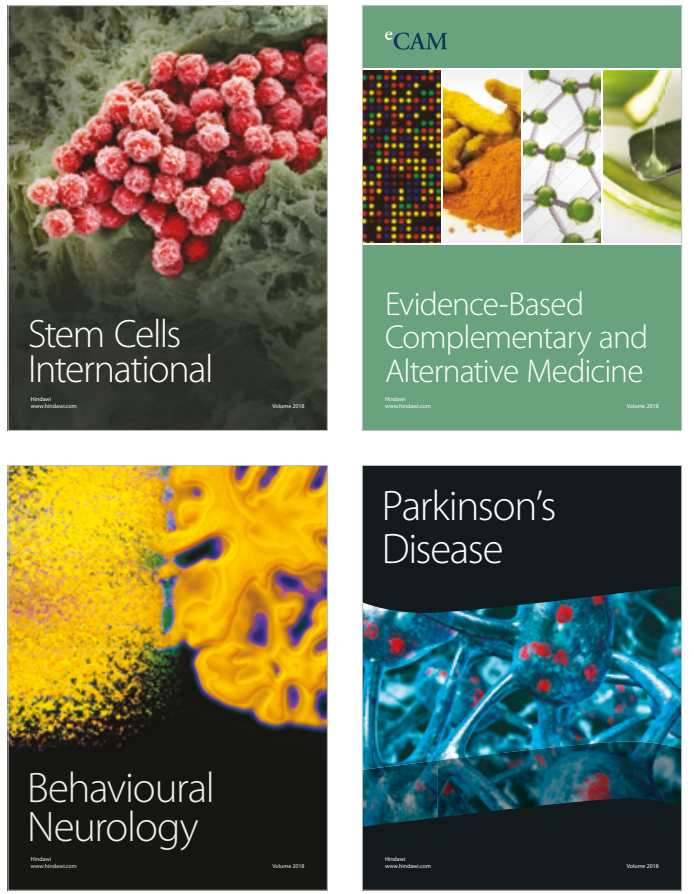

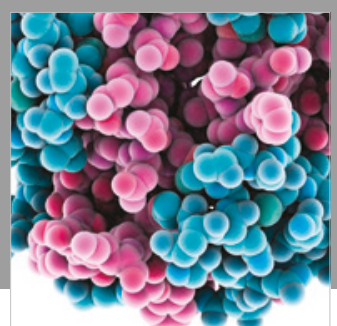

ournal of

Diabetes Research

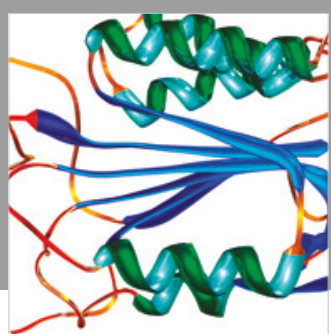

Disease Markers
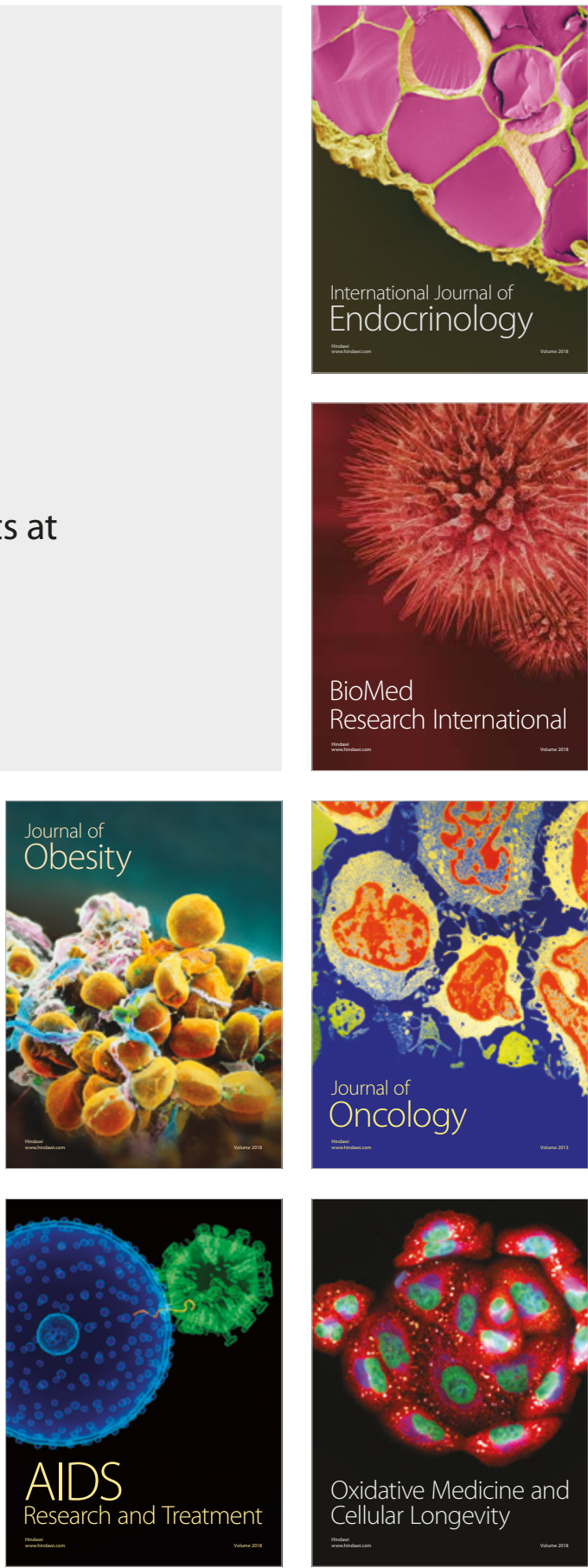